\title{
Context-Aware Query Processing in Ad-Hoc Environments of Peers
}

\author{
Nikolaos Folinas Panos Vassiliadis Evaggelia Pitoura Evangelos Papapetrou Apostolos Zarras \\ Department of Computer Science, \\ University of Ioannina, \\ 45110, Ioannina, Hellas \\ \{nfolinas,pvassi1,pitoura,epap,zarras\}@,cs.uoi.gr
}

\begin{abstract}
In this paper, we deal with context-aware query processing in ad-hoc peer-to-peer networks. Each peer in such an environment has a database over which users want to execute queries. This database involves (a) relations which are locally stored and (b) relations which are virtual or hybrid. In the case of virtual relations, all the tuples of the relation are collected from peers that are present in the network at the time when the query is posed. Hybrid relations involve both locally stored tuples and tuples collected from the network. The collaboration among peers is performed through web services. The integration of the external data, before they are locally collected to a peer's database is performed through a workflow of web service invocations. Summarizing the problem, due to the transitive nature of the extent of virtual relations, we cannot perform query processing in the traditional way, but rather, we have to involve contextaware query processing techniques that exploit the neighborhood of each peer and the web service infrastructure that deals with the heterogeneity of peers. To deal with the aforementioned problem we provide the following contributions. First, we formally define the system model. Next, we define $S Q L^{\mathrm{P}}$, an extension of traditional SQL on the basis of contextual environment requirements that concern the termination of queries, the failure of individual peers and the semantic characteristics of the peers of the network. In addition, we precisely define the semantics of $S Q L^{\mathrm{P}}$. We discuss issues of data integration, performed through workflows of web services. Moreover, we present a query execution algorithm as well as the formal definition of all the operators which take place in a query execution plan. Finally, we discuss issues of our prototype implementation.
\end{abstract}




\section{INTRODUCTION}

Nowadays, the synergy between network and database management systems provides opportunities for the integration and querying of various heterogeneous sources of information, spread over an ad hoc network of peers. The fundamental topic of this paper is the contextaware processing of queries in ad-boc networks of peers through web services. We assume the existence of a set of peers who communicate with each other, thus forming a time-varying ad hoc network of peers. For reasons of interoperability, we also assume that these peers use web services for their interactions. Each peer has a database where (a) data can be locally stored, or (b) descriptions of data are present, in a form that allows their collection from the appropriate peers and their subsequent querying with traditional database mechanisms. The querying and/or collection of these data is dependent on the state of the peer network and on the knowledge that the peer has about this state; therefore, each time a query is posed, its processing must be adapted to this state. In other words, the state of the peer posing a query and, most importantly, the state of its surrounding network constitutes the context under which the query is processed. Assume the case where several kinds of vehicles are driving in a highway. Each vehicle is a part of a global pervasive computing environment where computations can be performed, data can be exchanged between computing devices of the environment and information is interactively requested and presented to the users. Cars interact with each other through web services, providing dynamically changing information regarding the vehicle's location, velocity and fuel deposit. Moreover, each vehicle comprises services that offer static information concerning its type and technical characteristics. On the highway, there exist exits to parking areas, which may include facilities such as gas stations, fast food restaurants, medical help, and shopping centers. Each one of these facilities also comprises web services, which range from simple ones, reporting the existence of the facility, to more complex ones providing information regarding for instance the price lists, the availability of certain goods or the number of patients waiting for medical help. The users of the facilities of the pervasive environment, e.g., the drivers of the vehicles, can obtain information by posing queries to global information space of the environment. For instance, they may be interested in obtaining the information like the closest gas-station with a price of gasoline under $2 € /$ gallon, the closest Italian restaurant, or notifications for the average speed of all the cars ahead.

To facilitate the smooth operation of peers within the aforementioned environment, specific technical challenges must be addressed. A significant problem is the fact that traditional query 
processing must be reconsidered, to adapt to the particularities of our computing environment. In this paper, we are specifically interested in the problem of formally defining a declarative query language that enables the posing of queries over an ad hoc network of peers as well as the introduction of a mechanism for the transformation of declarative database queries to query execution plans.

First, we start with the theoretic formulation of the problem. We construct a directed graph of peers, where each node corresponds to a peer and each edge to the physical connection among two peers. The graph of peers is time-varying, since nodes and edges are added or invalidated as time passes. Apart from the possibility of communication, that dictates the structure of the graph, peers are further organized in communities, based on their semantic similarity, or classes, based on the interface of web services they support. All our deliberations are based on the principle of local scope, that dictates that no peer has a global knowledge of the entire graph, and therefore, all its decisions must be made depending solely on the knowledge that this node has at a given time point. Specifically, the viewpoint of a peer is the subset of the graph known to this peer at a given time point and the communities of the peer are sets of peers whose publicized characteristics fulfill a logical condition that classifies them into the appropriate community. The only classification that is not local is the class of each peer: we assume that a set of finite classes exists, each with an interface comprising a set of public web service operations that all class instances support. Every peer is created as an instance of one of the globally known classes.

With respect to the relationships among peers, each peer plays both the role of the server and the role of the client in this environment. As a server, the peer implements and exports the interface of web service operations prescribed by its class. The other peers of the system can invoke these web services at runtime. At the same time, the peer is responsible for answering queries posed by its users. In our framework, we discuss traditional database queries and therefore, the peer hosts a relational database where query processing takes place. The database includes different categories of relations. First, the database includes relations that obey the traditional assumption that a database hosts locally stored relations, whose extents are finite sets of locally stored tuples. In this paper, we extend this implicit assumption and assume that the extent of a relation can be spread among the peers forming the context of a peer. Therefore, only the description of the schema (or intention) of such a virtual relation is locally available, along with the description of the necessary web services that must be invoked in order to locally collect the relation's extent before continuing query processing as usually. This collection procedure practically dictates that a workflow of web services has to be executed for each peer of the viewpoint of the querying peer. 
Finally, a third category of relations involves hybrid relations, whose extent is partly locally stored and partly needs to be collected from the other peers.

The processing of queries in such an environment is inherently different to the traditional one. We have already mentioned the context-aware aspect of data collection for the population of virtual relations. Moreover, due to the volatile character of the state of the peer's graph, it is quite probable that the viewpoint of a peer is an inaccurate reflection of the state of the peer graph. In other words, it is quite possible that the graph has changed since the last refreshment of the viewpoint of a peer. In fact, the graph can possibly change also during the execution of a query; therefore, the processing of a query must be inherently designed to tolerate failures (i.e., web service invocations that do not respond) and continue operating regularly. Also, due to the possible vastness of the graph, it is necessary to be able to stop collecting answers after a certain, satisfactory amount of information has been collected. Based on these fundamental differences with traditional query processing, we introduce an extension of $S Q L, S Q L^{\mathrm{P}}$ that allows the user to exploit the context-dependent nature of the environment, by specifying the peers of interest though abstract criteria that involve their location in the graph, their community, their class, or QoS characteristics, like e.g., their availability. The usage of virtual tables is transparent in $S Q L^{\mathrm{P}}$. We exploit the previously introduced model to formally specify the semantics of $S Q L^{\mathrm{P}}$.

The processing of the queries in this extended version of SQL requires also an extension of the mechanism of query execution. Traditional relational database management systems translate the declarative SQL queries to procedural, executable plans that are expressed in the form of leftdeep trees of relational operators. Therefore, we introduce novel operators specifically tailored for the support of web service invocation and composition, in order to populate the virtual tables. Then, query processing can continue as usually. We have also implemented a mechanism that allows us to determine the necessary set of peers that are supposed to participate in a query and to visually display the produced plans to the user.

This paper is organized as follows: In Section 2, we propose $S Q L^{\mathrm{P}}$, an extension of SQL for adhoc P2P systems. To this end, we define a system model, we investigate language requirements and propose the syntax and semantics of $S Q L^{\mathrm{P}}$. In Section 3, we extend the relational algebra with novel operators and algorithms in order to map $S Q L^{\mathrm{P}}$ queries to query plans. In Section 4, we discuss implementation issues. Finally, in Section 5, we discuss related work and in Section 6, we conclude our results and discuss topics for future work. 


\section{SQL FOR PEERS: SYSTEM MODEL, REQUIREMENTS, SYNTAX AND SEMANTICS}

In this section, we formally define the system model. Then, we move on to formally define $S Q L^{\mathrm{P}}$, an extension of SQL for ad-hoc P2P systems.

\subsection{System Model}

A bird's eye view of the system infrastructure is modeled by a graph $G(V, E)$, comprising a set of nodes $V$ and a set of edges $E$ (Fig. 1). Each node in our system graph is a peer and each edge $e=$ $<u, v\rangle$ stands for the fact that node $u$ can communicate with node $v$. The notion 'can communicate' means that peer $u$ can send data or make a request for data to $v$ - in other words, the edge $\langle u, v\rangle$ implies that peer $u$ assumes (a) knowledge of existence and (b) network connectivity with node $v$. The edges are directed, in the sense that although node $u$ can communicate with $v$, the inverse does not hold (an edge $\langle v, u\rangle$ would be required to demonstrate such a fact). This is quite frequent in modern ad-hoc networks and deeply affects the design of efficient routing protocols (Abolhasan, Wysocki, \& Dutkiewicz, 2004). In the sequel, we will also refer to an edge between two nodes as a direct link. To discriminate between different nodes, each node is characterized by a globally unique identifier, peer $i d$.

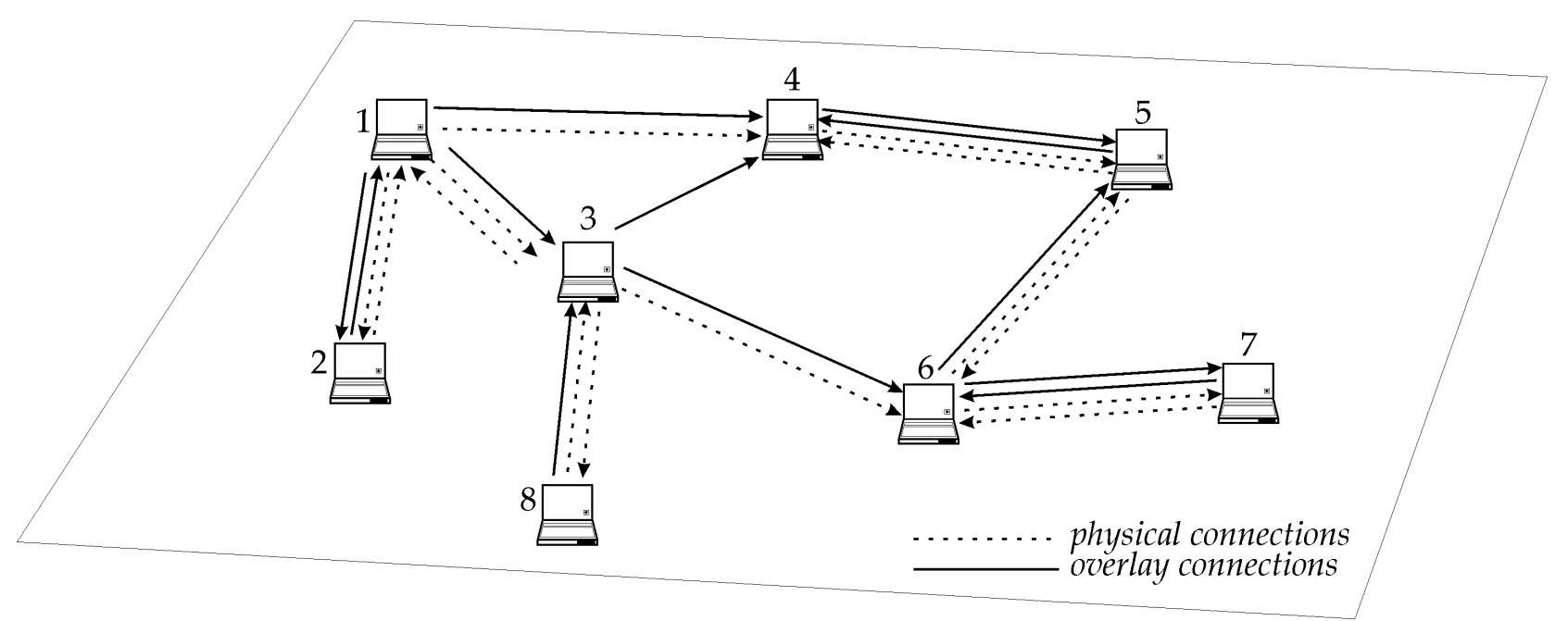

Fig. 1. A system's graph $G(V, E)$

As usually, a $\boldsymbol{p a t} \boldsymbol{b}$ between two nodes, say $u_{1}$ and $u_{2}$, is an acyclic sequence of consecutive edges belonging to $E$ that connects these two nodes. The distance of two nodes, say $u_{1}$ and $u_{2}$, is the cardinality of the minimum set of edges required to reach node $u_{2}$ through a path starting at $u_{1}$. In 
other words, the distance of two nodes is defined by the number of hops involved in the connecting path, which is a typical assumption in ad hoc networks research. We will denote the distance of two nodes as $\operatorname{distance}\left(u_{1}, u_{2}\right)$.

It is quite important, here, to stress the following properties of the system's graph:

- The graph is time-varying. In other words, nodes leave or enter the system as time passes. Furthermore, nodes move randomly, causing the destruction of existent links and the establishment of new ones.

- No node has a full knowledge of the system's graph, at any time point. On the contrary, it is important to design a system where each node has only a personal, restricted viewpoint of the graph. A fundamental principle in our deliberations is the locality of peer scope: each peer must be designed to operate by exploiting its own knowledge of a subset of the system, without counting on some higher-level authority to provide a global viewpoint of the system.

- It is also important that each node is designed to operate under the assumption that its knowledge of the graph is both incomplete and (possibly) inaccurate. This is a disadvantage related to the current networking technology for ad hoc networks (Chlamtac, Conti, \& Liu, 2003).

- The overall graph is not fully connected. In other words, it is not always possible to reach any node $v$ of $V$, starting from another node $u$.

Context $=$ Viewpoint of a node. At every time instant $T$, a node $u$ is aware of a subset of the system's graph, as it was configured at a previous time point $T \leq T$. This subset of the graph is called viewpoint of node $v$ at time $T$ and denoted by viempoint $(v, T)$. The subgraph viempoint $(v, T)$ is connected. This property is recursively defined as follows:

1. ve vieupoint $(v, T)$

2. All nodes $u$ that are connected to a node $x, x \in$ viempoint $(v, T)$ through an edge $(x, u)$ belong to viempoint $(v, T)$. In other words, first, all nodes $u$ that are connected to $v$ through an edge $(v, u)$ belong to viempoint $(\nu, T)$. Then, the nodes that can be reached from these ones are also added. This is recursively continued.

Inaccuracy is inherent in this definition. Firstly, all the knowledge about direct links refers to a time point $T$ in the past. This means that whatever changes have happened between $T$ and $T$ are obscure to $v$. The exact determination of time $T$ depends on the implemented routing protocol. Second, it is obvious that even if the overall set of nodes is finite (which, is not an assumption that we have made so far), it is clear that it is impractical or even impossible to maintain all the 
knowledge for the graph, for each node $v$. In fact this is the approach taken a large category of routing protocols known as on-demand routing protocols (Abolhasan et al., 2004).

Community. Apart from the physical connectivity among nodes, we can devise logical schemes for the connectivity of peers. In P2P terminology, the network of peers that share similar semantical properties is called an overlay network (Androutsellis-Theotokis \& Spinellis, 2004). In our setting, a community of nodes is a subset of $V$ who shares the same semantical properties. Each peer defines its own communities. Formally, semantical proximity is captured by a formula in a first-order predicate calculus. The principle of locality of a peer's scope imposes a design where each peer comprises a local set of communities, each defined as a subset of its viewpoint, upon fulfillment of the appropriate formula. Therefore, a community comm_name of a peer $u$ is defined as:

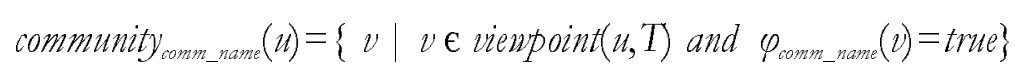

with $\varphi$ being a formula in a first-order predicate calculus that returns true or false given the properties of a node $v$.

Clearly, a node $u$ can have many communities and each node $v$ in the viewpoint of $u$ can belong to more than one communities of $u$. Moreover, assuming a simple community Unclassified that comprises all nodes that do not belong to any other community, the union of all communities of node $u$ returns viewpoint $(u, T)$, at a time point $T$. An interesting observation here is that if two or more nodes agree for a correspondence of communities, a P2P overlay is formed.

Web Services. Each node is equipped with a set of web service operations that it publishes, therefore giving the possibility to the rest of the nodes to invoke them. Formally, each node $u \mathrm{E}$ $V$ possesses a finite set of web service operations $W S^{\prime \prime}=\left\{w s^{u n}, w s^{m 2}, \ldots . ., n s^{u m}\right\}$ that are made public to the rest of the peers. In the sequel, we will not discriminate between the terms web service operations and web services.

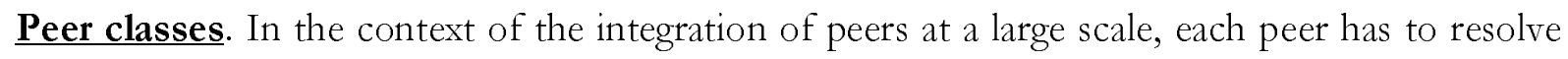
the problem of mapping the external interface of the other peers to its internal state. In other words, if a peer $u$ is to invoke a web service operation of another peer $v$, how does $u$ decide the mapping of the operation's parameters or the operation's result to its internal state? Typically, there are two well-known extremes from the database community to handle this problem, as well as intermediate solutions. 
- In the first extreme, a global schema is assumed. In distributed database systems (Ozsu \& Valduriez, 1991), a global schema is assumed for the whole environment and each local database comprises a subset of the global schema. This approach requires a universal common agreement over a global schema (and the implicit semantics hidden behind it). We find this requirement too restrictive for a large scale P2P environment that needs to be dynamically readjusted to novel peers that appear.

- An intermediate approach would be to hardcode all mappings among all peers. Still, this approach is to labor-intensive and clearly unable to scale up to the full extent of a P2P environment.

- In the second extreme, semi-automated techniques for schema matching have recently appeared in the literature. In the context of the schema mapping problem, where the mapping among two schemata must be discovered, semi-automated techniques have been proposed (Madhavan, Bernstein, Doan, \& Halevy, 2005). Nevertheless, a certain degree of training and supervision is required for a mapping to be derived and --to the best of our knowledge-- there is no fully automated, fast method for this purpose. Therefore, although this technology would resolve the scalability problem and the ad-hoc nature of the P2P environment, we cannot rely on its effectiveness for the moment.

To resolve the aforementioned problems of (a) scalability, (b) ad-hoc nature of the environment and (c) schema mapping discovery, we resort to an intermediate solution that provides a reasonable balance to all the aforementioned issues. We classify peers to peer classes, with the members of each class exporting the same web service operations. In other words, we assume a factory for each class, specifying the interface for each deployed instance.

We assume a traditional tree-based hierarchy of classes. Each subclass has a single superclass, whose interface it extends. All instances of the subclass are also instances of the superclass. Each node (a) directly belongs to exactly one class and (b) indirectly belongs to all the classes of the path that starts in the root and ends in its containing class in the tree of the class hierarchy. We call the set of nodes that directly belong to a class, immediate extent and the set of nodes that indirectly belong to a class (due to its subclasses) the extended extent. Classes that do not have any descendants are called base, or leaf classes. We denote the interface of a class $\mathrm{C}$ by interface $(C)$ and its immediate and extended extents as extent $(C)$ and $\operatorname{extent}_{\mathrm{e}}(C)$.

In Fig. 2 we can see the base classes VW, BMW, TOYOTA, SHELL, BP, HOTEL, RESTAURANT with their respective nodes. In Fig. 3 we can also observe the superclass CARS 
on top of the classes $V W, B M W$ and TOYOTA and a class GAS STATION as a superclass of SHELL and $B P$.

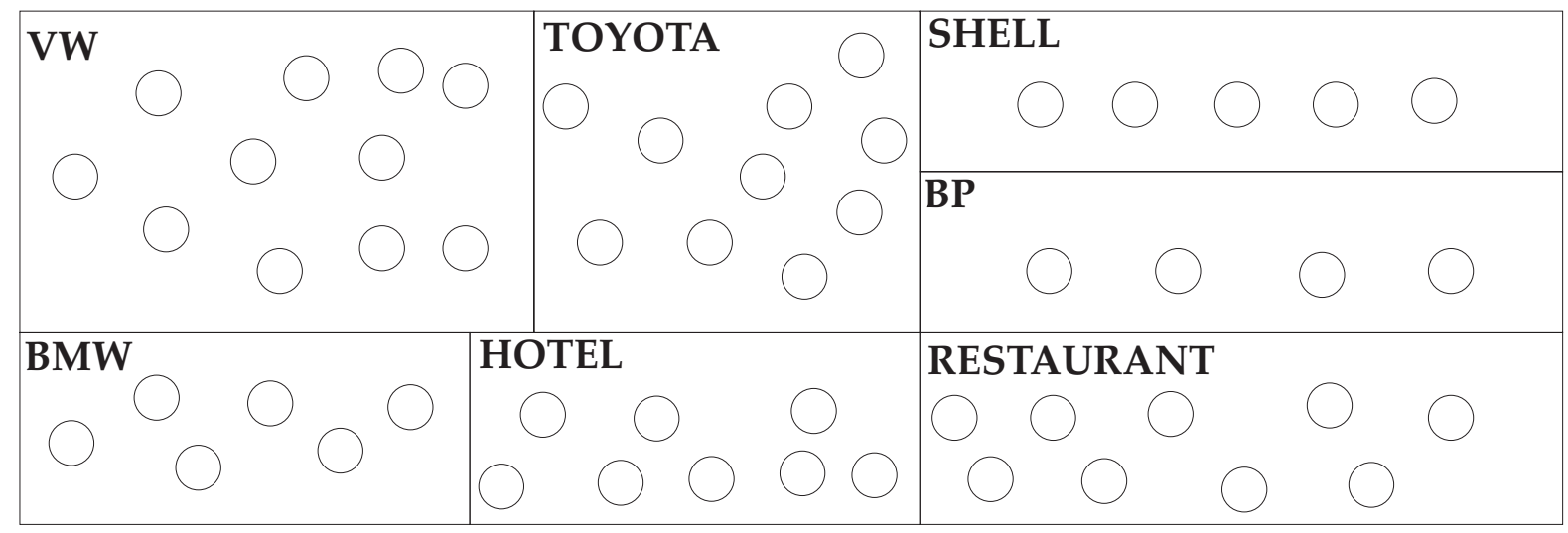

Fig. 2. Base classes with their corresponding nodes

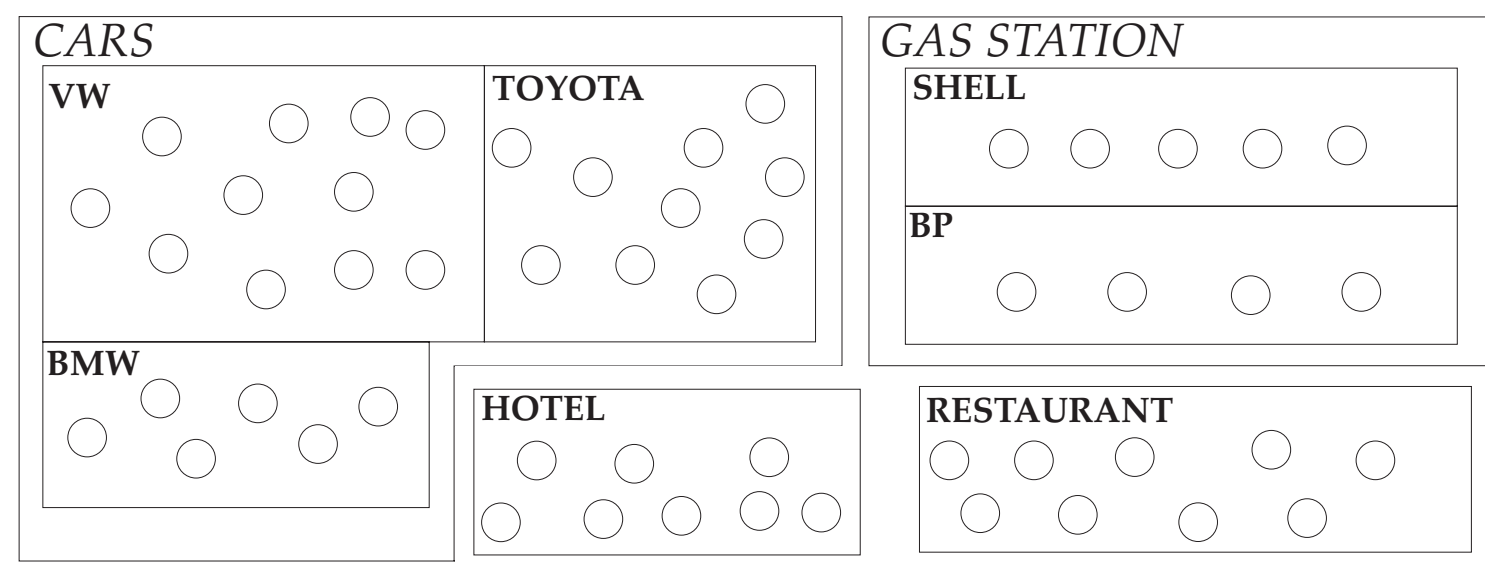

Fig. 3. A hierarchy of classes with their corresponding nodes

The aforementioned problems of integration are resolved in a balanced fashion. With respect to the scale-up of the environment, the integration problem is only dependent on the number of peer classes and not on the number of their instances. Although we anticipate a reasonably small number of peer classes, still the problem of integration is present. We assume a hard-coded, intermediate solution between pairs of classes. This does not necessarily require that all classes are mapped to each other; the only effect of the absence of a mapping would be that two instances belonging to non-reconciled classes cannot query each other, without a total failure of the system. Moreover, it is straightforward to devise mechanisms for incremental updates of class mappings for the deployed instances, so that, as new classes are added and the interfaces of old classes are updated, the deployed instances are informed on the new situation. With respect to the ad-hoc nature of the P2P environment, the problem of class integration is orthogonal and not affected. The last problem, discovery of schema mappings is resolved at the factory level 
(although we recognize that we still need the same amount of coding effort as in traditional mediator-wrapper environments).

Difference between classes and communities. The class of a node is an inherent property of the node, determined once and for all at the creation of the node, mainly for integration purposes, whereas the community (or communities) to which it belongs is a potentially timevarying property that is determined individually by the other peers and is mainly used for querying purposes.

Clock. Each peer has its own clock. The clocks of the peers are not necessarily synchronized.

Peer database. Each peer has a database, which comprises a set of relations. Each relation has a schema or intention comprised of a finite set of distinct attribute names. Also, each relation has an extension which is a finite subset of the Cartesian product of the domains of the attributes of the relation's schema. The relations of a peer's database are classified in the following categories:

- Locally stored (or local) relations: Local relations are relations whose extension involves tuples that are locally stored at the peer that carries the relations' database. In other words, local relations are exactly the same as in traditional relational databases.

- Virtual relations: Virtual relations are relations whose schema is fixed and locally known, but whose extension is not locally stored in the database of the peer. On the contrary, the extension of a virtual relation is collected from the appropriate peers at query time. Practically, this means that each time a user poses a query involving a virtual relation, the peer determines the set of peers who are to be contacted (along with the appropriate sequence of web service operations of these peers that are to be invoked), collects the respective tuples, transforms them to the schema of the virtual relations, and, finally, stores (or "materializes") them. Then, query processing can be performed as usual.

- Hybrid relations: Hybrid relations are variants whose extension includes both locally stored tuples and tuples to be collected from other peers.

Each tuple collected for a relation belonging to the last two categories is tagged with a timestamp, produced by the clock of the node that receives the incoming tuple. The timestamp corresponds to the transaction time of the tuple, i.e., the exact time point of its entrance to the receiver's database. A tuple's timestamp will be used for caching purposes. 
Peer Characteristics. Each peer is characterized by several properties that can either be determined by the peer itself or by the class to which it belongs. Specifically, the characteristics that we adopt are:

- (Average) Availability, i.e., the probability that the peer is operational at a given time instant.

- (Average) Response Time, i.e., the average time needed for a web service operation of the peer to execute.

Peer's System Catalog. Each node u needs a system catalog for its proper operation. The catalog includes useful information about the nodes known to $u$. Specifically, this information refers to:

- Class of the other nodes.

- Communities of the other nodes.

- Distance from other nodes.

- Node characteristics, like availability, and response time.

\subsection{Results Collection from Other Peers}

In this subsection we discuss issues of tuple collection for the virtual and hybrid relations. First, we formally introduce workflows of web service operations. Next we discuss how the mapping of the workflow's result to a peer's relation is performed and finally, we formalize issues of result materialization.

Workflow $w f^{u . R}\left(\boldsymbol{u}_{i}\right)$. Assume a peer $u$ that poses a query and invokes web service operations from a set of peers $u_{1}, u_{2}, \ldots . ., u_{z}$ in order to collect their tuples. In principle, it is quite possible that the requested information from a certain peer can only be obtained after the invocation of a workflow of web service operations (rather than a single operation). For example, assume that a peer using the European metric system collects the velocities of other peers of class $C A R$, and a certain class of cars returns miles instead of kilometers. The conversion can be performed through a simple BPEL workflow. We denote each of these workflows as $w^{f^{R}}\left(u_{i}\right)$, with $1 \leq i \leq z$ : Each such workflow $w$ is an acyclic directed graph $G_{w}\left(V_{w} E_{w}\right)$, with operations being modeled as nodes and edges being the representatives of control passing. Edges are tagged with the conditions under which they are fired at runtime. Each workflow has also a flat relational schema, comprising a set of attributes that result from the possible un-nesting of the XML elements of 
the final message delivered by the workflow. Finally, the workflow has an extension, dynamically created at runtime, that instantiates the aforementioned schema.

Mapping of other peers' web services to virtual relations. In this paragraph, we formally discuss the mechanism that allows peers to collect tuples from the peers of their viewpoint. Assume a peer $\mathrm{u}$ that poses a query and invokes web service operations from a set of peers $u_{1}$, $u_{2}, \ldots, u_{q}$ in order to collect their tuples. The application of the workflow $w f^{R}\left(u_{i}\right)$ results to a set of tuples under the schema $\left(B_{1}, B_{2}, \ldots, B_{m}\right)$, possibly after a set of XML un-nesting operations. Assume $\mathrm{R}\left(A_{1}, A_{2}, \ldots, A_{n}\right)$ to be the schema of $\mathrm{R}$, the mapping between the two schemata is a function $f_{\text {mas }}$, with $f_{\text {mas }}:\left(A_{1}, A_{2}, \ldots, A_{n}\right) \times\left(B_{1}, B_{2}, \ldots, B_{m}\right) \rightarrow\{$ true, false $\}$. We impose the constraint that for each $A_{i}, 1 \leq i \leq n$, there exists at most one $\mathrm{B}_{j}, 1 \leq j \leq m$, to which $A_{i}$ is mapped. As usually, all attributes of the workflow schema that are not mapped to the schema of the target relation are projected-out, whereas all the relation's attributes that are not populated by the workflow are filled with NULL values. The following example clarifies the aforementioned process. Assume the relation $\mathrm{R}\left(E \_I D, E \_S A L A R Y, E \_A G E\right)$ in the database of node $u$ and let the workflow that is mapped to $\mathrm{R}$ for node $v$ have the schema (ID,AGE,NAME). The workflow provides no information on salaries and the database does not store any data on names. Therefore, our mappings resulting to true are:

$f_{\text {mas }}\left(E \_I D, I D\right)=$ true, $f_{\text {mat }}\left(E \_A G E, A G E\right)=$ true, with the rest of all the other possible mappings of the Cartesian product of the two schema being evaluated to false. The transformation at an instance level is simple: (a) we project-out all unnecessary workflow attributes, (b) we introduce NULL-valued attributes for the relation's attributes for which no workflow attribute exists, (c) we appropriately re-order the attributes of the workflow schema to match the relations attributes and (d) we populate the target table.

Full-Partial materialization. Whenever a workflow is executed for a certain peer and the produced results are successfully stored at the extent of the target virtual relation, we say that we have materialized these results. The fact that the results of a certain workflow for peer $u_{i}$ have been materialized at the relation $\mathrm{R}$ of peer $u$ is denoted as $\left(w f^{R}\left(u_{i}\right)\right)$. Full materialization for a relation $R$ of a peer $u$ is the state of a query when all workflows for all the peers that have been selected to populate $\mathrm{R}$ have been successfully executed. We denote full materialization by $M(u \cdot \mathrm{R})$. Assuming $V^{\text {zall }}$ be the set of these identified peers, we can formally define full materialization as $M(u \cdot R)=U\left(u f^{R}\left(u_{i}\right)\right)$, with $u_{i} \in V^{\text {adl }}$.

Partial materialization for a relation $R$ of a peer $u$ is the state of a query when the workflows for a clean subset of the peers that have been selected to populate $R$ have been successfully 
executed. We denote partial materialization by $M^{b}(u \cdot R)$. Assuming $V^{\text {all }}$ be the set of the peers that have been selected to participate in the population of $R$, and $V^{\lambda}$ be the set of the peers whose results have been successfully materialized, we can formally define partial materialization as

$$
M(u \cdot R)=U\left(w f^{R}\left(u_{i}\right)\right) \text {, with } u_{i} \in V^{i}, V^{i} \subset V^{\text {all }} .
$$

\section{3 $S Q L^{P}$ : an Extension of SQL for Ad-Hoc P2P Networks}

In this section, we discuss the extension of SQL that we introduce. The proposed language $S Q L^{\mathrm{P}}$ (SQL for Peers) implements all the aforementioned requirements. Figure 4 presents the general structure of an $S Q L^{\mathrm{P}}$ query. We use [...] to refer to optional parts of the language and the expression *AND/OR* to signify that different clauses can be connected through one of these logical connectors.

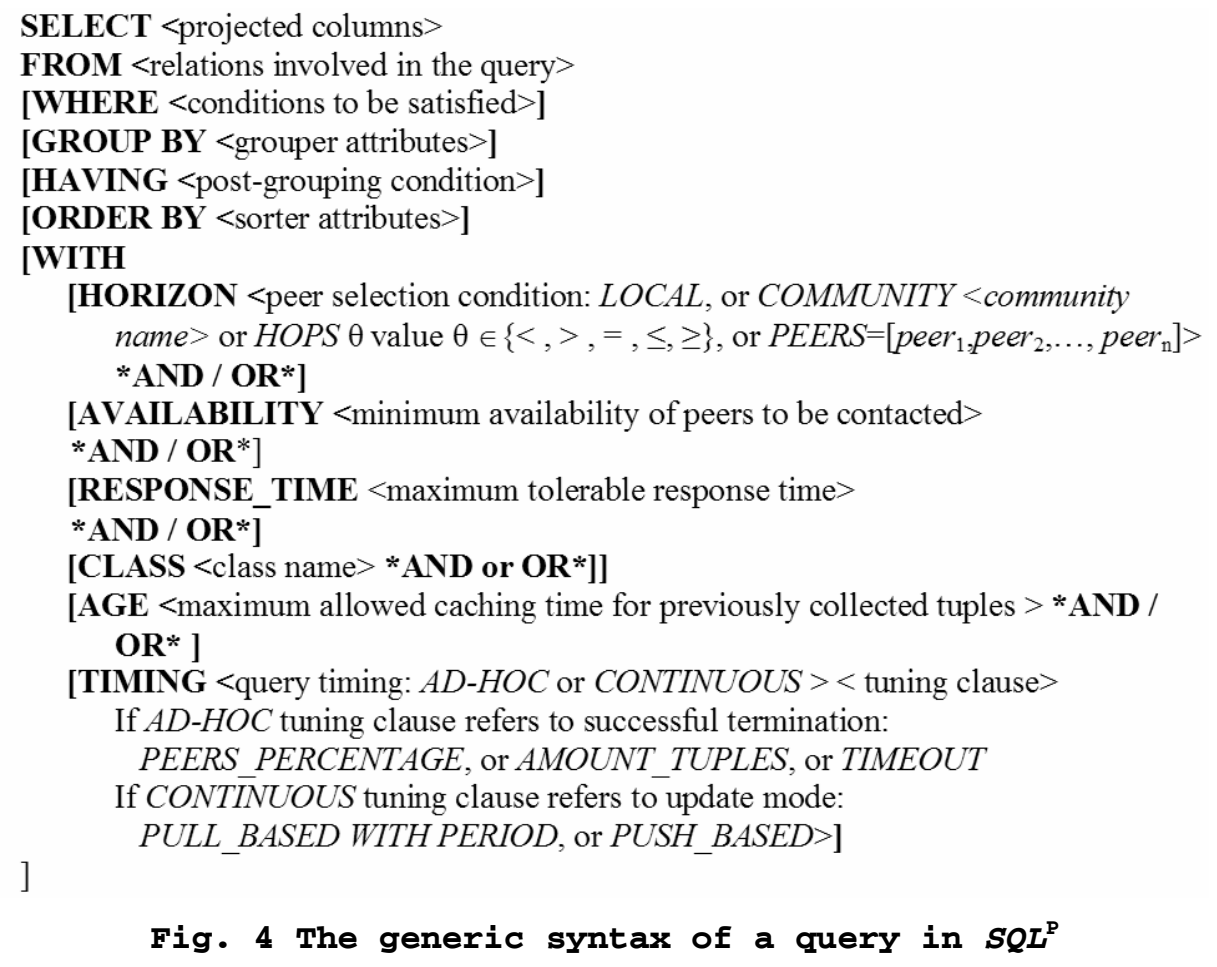

Querying the graph of peers. Assume a query 2 submitted at node $u$ at the time point $T$. Let $\left\{R_{1}, R_{2}, \ldots, R_{n}\right\}$ be the relations that participate in the FROM clause of the query. Then, we can write the query as: $Q\left(R_{1}, R_{2}, \ldots, R_{n}\right)$. Without loss of generality, we can assume that the first $k$ relations $R_{1}, R_{2}, \ldots, R_{k}, k \leq n$, are virtual or hybrid. In order to be able to define the semantics of the query properly we need to materialize these relations and then, execute the query over their 
collected extent as usually. Nevertheless, before specifying this semantics, we need to define the following concepts.

Peers of Interest. The query 2 , posed over peer $u$ is divided in three parts. The first part is composed of the traditional SQL clauses, the second part comprises the clauses of our extension that occur after the keyword WITH that have the purpose of determining which peers are to be contacted, and the third part concerns the timing of the query.

The second part of the query depends on criteria like the horizon of the query of the graph of the viewpoint of peer $u$ (HORIZON), QoS characteristics (AVAILABILITY, RESPONSE_TIME), the class of the peers (CLASS) and the age of the stored tuples in the virtual relations (i.e., if a peer has been recently contacted, as specified by the AGE clause, it is not necessary to contact it again). Remember that due to the nature of the interaction among peers, it is not feasible to simply broadcast a request for tuples; on the contrary, specific web service operations must be invoked on the specific port types of the peers.

In terms of semantics, we divide the second part into atomic conditions, logically connected through the connectors AND and OR. Assuming that these atomic conditions are $C_{1}, C_{2}, \ldots, C_{n}$ the non-traditional part of the query can be rewritten in a disjunctive normal form, i.e., a disjunction of conjunctive conditions.

The interesting aspect of this part is that a preparatory query must be performed over the system catalog to determine specifically which peers must be contacted in order to materialize the virtual relations. Contacting a peer means that for each virtual/hybrid relation in the FROM clause of the query the execution of the appropriate workflow must be initiated. In terms of semantics, each atomic condition specifies a set of peers of the viewpoint of $u$ that qualify to be contacted. Given an atomic condition $C$, we define the set of peers of interest $V^{\prime \prime}(C)$ to be the set of peers that belong to the catalog of peer $u$ that fulfill C. Specifically, given a time point $T$ for a query $\mathcal{Q}$ containing $C$,

$$
V^{\mu}(C)=\{\nu \mid v \in \operatorname{viewpoint}(u, T): C(\nu)=\text { true }\}
$$

We do not involve timepoint $T$ to avoid overloading the notation. Having defined the peers of interest for an atomic condition, it is straightforward to obtain the set of peers of a composite condition in disjunctive normal form: The intersection of the peers of interest of the atomic conditions produces the peer sets of each conjunct; these sets are subsequently ORed to produce the final set of peers of interest of the query, which are to be contacted.

Now, we are ready to define the semantics of each individual clause concerning the determination of the peers of interest. 
HORIZON: The condition of the HORIZON clause determines the peers of interest on the basis of the position in the graph, or their semantical characteristics. The clause allows several possibilities to the users. Assuming that the condition of the HORIZON clause is $C_{1}$, and $V H^{\prime \prime}\left(C_{1}\right)$ is the resulting set of peers of interest, we can specify $V H^{u}\left(C_{1}\right)$ for each of the following possibilities that $S Q L^{\mathrm{P}}$ offers:

1. The only peer of interest is the local querying peer $\left(C_{1}\right.$ : LOCAL)

$$
V H^{u}\left(C_{1}\right)=\{u\}
$$

2. The peers of interest are the ones of a certain community of the peer $\left(C_{1}:\right.$ COMMUNITY $<C_{-} N A M E>$ )

$$
V H^{u}\left(C_{1}\right)=\left\{v \mid v \mathrm{e} \text { viempoint }(u, T): v \mathrm{e} \text { community }\left(C_{-} N A M E, u\right)\right\}
$$

3. A radius of a certain number of hops dictates the peers of interest $\left(C_{1}:\right.$ HOPS $\theta$ value, with $\theta \mathrm{e}$ $\{=,<, \leq,>, \geq\})$

$$
V H^{u}\left(C_{1}\right)=\{\nu \mid v \in \text { viempoint }(u, T): \text { distance }(u, \nu) \theta \text { value, with } \theta \in\{=,<, \leq,>, \geq\}\}
$$

4. A set of peer id's, i.e., a set of specifically requested peers, determines the peers of interest

$$
\begin{aligned}
\left(C_{1}: \text { PEER } S=\right. & \left.\left\{\text { peer }_{1}, \text { peer }_{2}, \ldots, \text { peer }_{n}\right\}\right) \\
& V H^{u}\left(C_{1}\right)=\left\{v \mid v \in \operatorname{vieupoint}(u, T): v \in\left\{\text { peer }_{1}, \text { peer }_{2}, \ldots, \text { peer }_{n}\right\}\right\}
\end{aligned}
$$

All the necessary information for the evaluation of any of the aforementioned atomic conditions is found in the system catalog of $u$.

Quality of Service: The clauses concerning the AVAILABILITY and RESPONSE TIME of the peers of interest aim to guarantee a certain level of quality of service for the peer posing a query.

CLASS: It is possible that we only need to query the peers of a certain class. Classes carry both structural typing information (as they statically define the interface of their instances), but also semantic information (as collections of semantically -therefore structurally- similar instances). In $S Q L^{\mathrm{P}}$, it is easy to specify an atomic condition that restricts the peers of interest to a certain class, by giving a condition of the form $C_{4}: C L A S S=$ class_name. Assuming $V C^{u}\left(C_{4}\right)$ the result set of peers of interest, and class ( 2$)$ a function that returns the class of each peer from the system catalog of the querying peer, the resulting set of peers of interest is formally defined as:

$$
V C^{u}\left(C_{4}\right)=\{\nu \mid v \text { vieupoint }(u, T): \text { class }(v)=\text { class_name }\}
$$

AGE. Apart from the constraining of peers where their properties are taken as criteria for their inclusion in the resulting set of peers of interest, we can perform some form of caching in the 
extents of the collected tuples for virtual or hybrid relations. In other words, assuming that a peer is frequently queried, it is not obligatory to pay the price of invoking its web service operations, executing the data transformation workflow and materializing the same results again and again, but rather, it is resource efficient to cache its previous results. The AGE clause of $S Q L^{\mathrm{P}}$ provides the possibility of specifying a maximum caching age for incoming tuples in a virtual/hybrid relation.

Query timing. Having clarified the general mechanism for the determination of peers of interest, we move on to provide the specification for the timing of queries. Fundamentally, we have two modes of operation: ad boc or continuous. Each mode has its own tuning parameters.

- If the query is continuous, this means that the user is continuously notified on the status of the query result.

- If the query is ad-hoc, the query eventually has to terminate. Differently from traditional query processing (which operates on finite sets of always available, locally stored tuples), we need to tune the conditions that signify termination of a query that has been late to complete its operation, either due to peer failures, or the size of the peers graph. To capture these exceptions, we can terminate a query upon (a) the completion of a timeout period of execution, (b) the materialization of a certain amount of tuples, that the user judges as satisfactory for his information, or (c) the collection of responses from a certain percentage of peers that were initially contacted. In all these cases, the execution of the workflows whose results have not been materialized is interrupted, the rest of the query is executed as usually and the user is presented with a partial --still, non-empty-- answer.

Query Execution. At this point we can describe the exact set of steps for executing a query. Suppose that at random time $T$, a query $Q$ is performed by node $u$. Let $\left\{R_{1}, R_{2}, \ldots, R_{n}\right\}$ be the relations involved in query $Q$. Then the query can be written in the form: $Q\left(R_{1}, R_{2}, \ldots, R_{n}\right)$. We can assume that the relations $R_{1}, R_{2}, \ldots, R_{k}$, with $k \leq n$ are virtual or hybrid, without any impact on the generality. All tables $R_{1}, R_{2}, \ldots, R_{k}$ must be filled with tuples. The procedure is the same for all tables, therefore we will present it only for table $R_{1}$.

The first step is to determine the set of target peers for node $u$ that performs the query $\left(V^{n}(C)\right.$ ), by evaluating $C$ over the set of peers belonging the viewpoint of $u$ (viempoint $(u)$ ). $C$ comprises of the conditions located at the clauses AGE, HORIZON, AVAILABILITY, RESPONSE_TIME and CLASS. 
Let $V^{\mu}(C)=\left\{\mathrm{u}_{1}, \mathrm{u}_{2}, \ldots, \mathrm{u}_{\mathrm{m}}\right\}$. For each node $V^{n}(C)$ the appropriate web services are invoked in order to require the appropriate tuples. Let also $u f^{R^{1}}\left(u_{1}\right), w f^{R^{R}}\left(u_{2}\right), \ldots, w f^{R^{1}}\left(u_{m}\right)$, be the appropriate workflows of the peers belonging to $V^{\pi}(C)$.

The schema of each workflow is matched to the schema of relation $R_{1}$, which is the target relation. In the following, the clause TIMING is evaluated to determine the execution mode of the query (continuous or ad-hoc) and the completion condition of the query. The next step is to attempt the execution of $w f^{R 1}\left(u_{i}\right) \cdot\left(\left(w f^{R 1}\left(u_{i}\right)\right)\right)$ and then perform a full or partial materialization of $\mathrm{R}$, which is located in $u$, according to the query completion condition which was mentioned before. Table $R_{1}$ is populated with the appropriate tuples and is ready to be queried. The same procedure is performed for all other virtual or hybrid tables. Therefore all tables of $u$ are ready to be queried. At this point the query of $u$ is performed over tables $R_{1}, R_{2}, \ldots, R_{n}$ based on traditional database methodology.

\subsection{Examples}

In the rest of this section, we will present examples of $S Q L^{\mathrm{P}}$. Assume a peer network of the topology of Fig. 5, consisting of 5 peers, each representing a car in the highway. Queries are posed to peer $p_{1}$, that classifies the rest of the peers in two communities, (a) the community of dark shaded close peers (Distance_Under_5 $\mathbf{k m}$ ) and (b) the community of light-shaded, distant peers (Distance_Over_5km). Peer $p$, is informed on the existence and connectivity of the rest of the peers through the underlying routing protocol that operates as a black box in our setting.

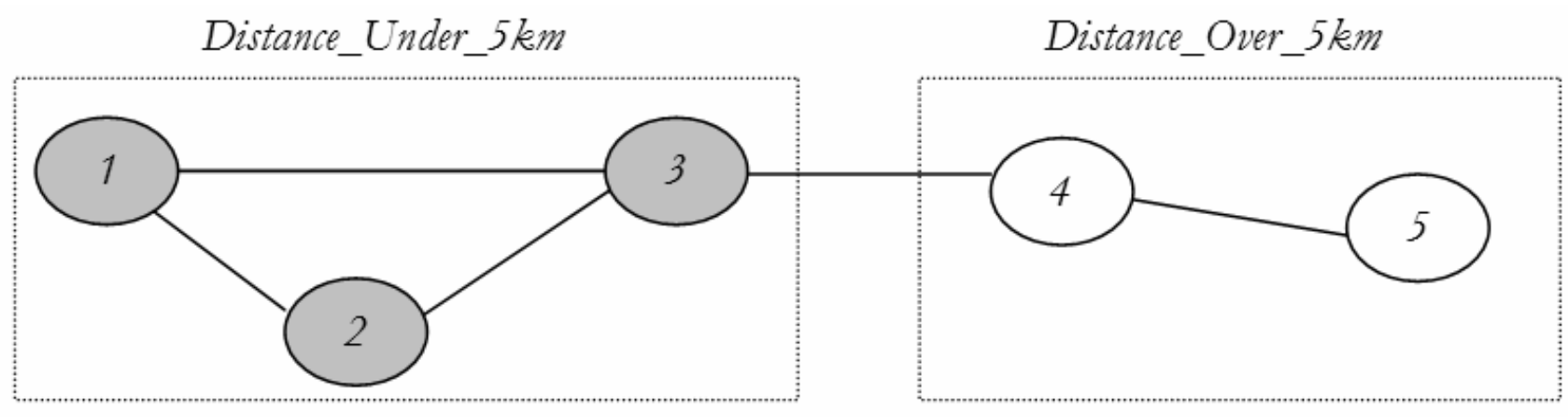

Fig. 5. Graph configuration for query posing

Peer $p$, carries a database consisting of two relations with the following schemata:

CARS(ID, PLATE, BRAND, VEL)

BRANDS( $\underline{B R A N D}$, COUNTRY, METRIC_SYSTEM) 
The first relation describes the information collected from the peers contacted (and mainly serves queries about the velocity of the cars in the context of the querying peer). This relation CARS is virtual: each time a query is posed, tuples must be collected from the context of peer $p_{1}$ to populate it. The attribute BRAND is a foreign key to the relation BRANDS that is static and locally stored. Primary keys are underlined and the semantics of the attributes are the obvious ones. In the sequel, we give examples of $S Q L^{\mathrm{P}}$ queries over the abovementioned environment.

Example 1. By this example we illustrate different situations where we can determine the peer nodes to which the query is addressed. Different strategies may be used for choosing the peers to query. In any case the decision is based on characteristics of the peers such as availability, response time, class of web services implemented, etc. Peer $p_{i}$ wishes to know the license number, velocity and manufacturing country of all cars belonging to its community. Furthermore, the peer that poses the query wishes to limit it to those peers that: (a) are located no more than 5 Km away (Distance_Under_5km), (b) their availability is more than $60 \%$, (c) their response time is less than 4 secs and finally, (d) implement the European class of Web Services. The syntax of the examined query is depicted in Fig. 6.

Example 2. Peer $p$, wishes to know the license number, velocity and manufacturing country of all cars. The peer also wishes to complete the query when more than $70 \%$ percent of the target peers have replies successfully (Fig. 7). To determine the target peers, the requesting peer selects the peers based on its catalog and according to their response time. The execution of the query stops when the requested percentage of $70 \%$ in our case is satisfied.

Example 3. Peer $p$, wishes to know the license number, velocity and manufacturing country of all cars. The peer also wishes to complete the query when more than 5 tuples have been collected for the relation CARS (Fig. 8). The requesting peer contacts each peer that appears in its catalog. This procedure ends when the count of currently collected tuples becomes greater or equal to the posed limit.

Example 4. Peer $p$, wishes to know the license number, velocity and manufacturing country of all cars. The peer also wishes to complete the query within a timeout of $7 \mathrm{sec}$ (Fig. 9). The requesting peer contacts each peer that appears in its catalog. This procedure ends when the timeout is reached. 
SELECT CARS.PLATE,CARS.VEL,BRANDS.COUNTRY

FROM CARS,BRANDS

WHERE CARS.BRAND=BRANDS.BRAND

WITH

HORIZON COMMUNITY Distance_Under_5km AND

AVAILABILITY $>60 \%$ AND

RESPONSE_TIME $<4.0$ AND

CLASS $=$ 'european'

Fig. 6. Query 1

SELECT CARS.PLATE,CARS.VEL,BRANDS.COUNTRY

FROM CARS,BRANDS

WHERE CARS.BRAND=BRANDS.BRAND

WITH

TIMING AD-HOC PEERS_PERCENTAGE $>70 \%$

Fig. 7. Query 2

SELECT CARS.PLATE,CARS.VEL,BRANDS.COUNTRY

FROM CARS,BRANDS

WHERE CARS.BRAND $=$ BRANDS.BRAND

WITH

TIMING AD-HOC AMOUNT_TUPLES $>5$

Fig. 8. Query 3

\author{
SELECT CARS.PLATE,CARS.VEL,BRANDS.COUNTRY \\ FROM CARS,BRANDS \\ WHERE CARS.BRAND $=$ BRANDS.BRAND \\ WITH \\ TIMING AD-HOC TIMEOUT $>7$
}

Fig. 9. Query 4

\title{
3 QUERY PROCESSING FOR $S Q L^{\mathrm{P}}$ QUERIES
}

In this section, we deal with the problem of mapping the declarative $S Q L^{\mathrm{P}}$ queries to executable query plans. As already mentioned, the execution of traditional SQL queries relies on their mapping to left-deep trees whose leaves are database relations, internal nodes are operators of the relational algebra and edges signify pipeline of the results of a node to another. Clearly, since we raise fundamental assumptions of traditional database querying, such as the finiteness and locality of tuples as well as the conditions under which a query terminates, we need to extend both the set of operators that take part in a query and the way the query tree is constructed. In this section, 
we start by introducing the novel operators for query processing. Next, we discuss how we algorithmically determine the set of peers of interest and finally, we discuss the execution of a query.

\subsection{Novel Operators}

In this subsection, we start with the operators that participate in $S Q L^{\mathrm{P}}$ query plans. We directly adopt the Project, Select, Group, Order, Union, Intersection, Difference and Join operators from traditional relational algebra and move on to define new operators. First, we discuss operators that are used to construct the set of peers of interest. Then, we present the operators that actually take part in a query plan.

\section{Operators applicable to the catalog of a peer:}

- Check Tables: operator Check_Tables determines whether the tables belonging to the FROM clause of a query are virtual, hybrid or local. The input to the operator is the FROM clause of the query and the output is the same list of tables, each annotated with the category to which it belongs.

- Check Peers: This is a composite operator that applies the procedure mentioned in Section 2 for the determination of a set of peers out of a condition in disjunctive normal form. All clauses of the form HORIZON, AVAILABILITY, RESPONSE_TIME and CLASS are evaluated over the catalog through a Check_Peers operator and the set of peers of interest is determined by combining the results of these operators through the appropriate Unions and Intersections.

- Check Age: The Check_Age operator is also an operator used to determine the set of peers of interest. For each relation that hosts transaction time and producing peer attributes, an invocation of the Check_Age operator scans the extent of the relation, and identifies the appropriate tuples and their peers. The output is passed to the appropriate Difference operator that subtracts the identified peers from the previously determined set of peers of interest.

\section{Operators that participate in query plans:}

- Call WS: This operator is responsible for dynamically determining which web service operation, over which port type of a specific peer must be invoked. Each web service of a 
peer to be invoked is practically wrapped by this operator. The result is collected and forwarded to the operator managing the execution of a workflow of web services.

- Wrapper Pop: This operator is used in order to support the monitoring and execution of the workflow of web services that populate a virtual or hybrid table. For each peer contacted in order to populate a certain virtual/hybrid relation, a Wrapper_Pop operator is introduced. Once the final XML result has been computed, its tuples are transformed to the schema of the target relation.

- Fill: A Fill operator is introduced for each virtual relation. The operator takes as input all the results of the underlying Wrapper_Pop operators (one for each peer of interest) and coordinates their materialization. Also, Fill checks the necessary conditions concerning the timing and termination of the query and, whenever termination is required, it signals its populating operators appropriately.

- ExAg (Execute Again): This operator is useful only in continuous queries and practically restarts query execution whenever the query period is completed.

\subsection{Construction of the Query Tree}

In this paragraph we discuss a simple algorithm to generate the tree of the query plan. Assume that a query is posed to peer $p_{1}$ and its viewpoint comprises $n$ peers, specifically $p_{1}, p_{2}, \ldots, p_{n}$. The algorithm for the construction of the query tree is a bottom up algorithm that builds the tree from the leaves to the top and is described as follows:

1. We discover the virtual or hybrid relations that participate in the query. A specific sub-tree will be constructed for each of them.

2. We determine the set of peers of interest. For each peer that participates in the population of a certain relation, the leaves of the respective sub-tree are nodes representing the peer to be contacted. To keep the tree-like form of the plan, each peer can be replicated in each sub-tree to which it participates; nevertheless, each peer can also be modeled by a single node without any significant impact to the execution of the query.

3. We introduce a Wrapper_Pop for each peer that coordinates all the Call_WS operators that pertain to the operations of the peer. Between the peer node and the Wrapper_Pop, we introduce the appropriate Call_WS operators.

4. For each virtual or hybrid relations we introduce a Fill operator that combines the output of all the respective Wrapper_Pop operators; therefore it is their immediate anscestor. 
5. Having introduced the Fill operators, the virtual or hybrid relations can be materialized and act as local ones. Therefore, the rest of the query tree is built as in traditional query processing.

6. If the query is continuous, we add an appropriate $\boldsymbol{E x A g}$ operator at the top.

\subsection{Execution of a Query though the Query Tree}

The execution of the query follows a simple strategy. First, we materialize the virtual / hybrid relations. Then, we execute the query as usual. Clearly, although this is not the best possible strategy for all cases (esp., when only non-blocking operators are involved), we find that performing further optimizations is an orthogonal problem, already dealt in the context of blocking operators for streaming data (Babcock et al., 2002). Therefore, in this paper we consider only this baseline strategy since all relevant results can directly be introduced in the optimizer module of a peer. Specifically, the set of steps to follow for the execution of the query are:

1. All the Call_WS operators are activated and the appropriate services are invoked.

2. The Wrapper_Pop operators collect the incoming XML results and queue them towards the appropriate Fill operators that further push them towards the extents of the relations in the hard disk. This is performed in a pipelined fashion.

3. Once all virtual/hybrid relations have been materialized, the rest of the query plan is a traditional left-deep tree that executes as usually.

\subsection{Example}

In the following, we discuss the construction of the query plan for the query of Fig. 10.

SELECT CARS.PLATE,CARS.VEL,BRANDS.COUNTRY

FROM CARS,BRANDS

WHERE CARS.BRAND=BRANDS.BRAND

WITH

AGE $<5$ AND

HORIZON COMMUNITY DISTANCE UNDER 5KM AND

TIMING CONTINUOUS PULL_BASED_PERIOD $=7$ AND

AVAILABILITY > 60\% AND

RESPONSE_TIME $<3.0$ AND

CLASS $=$ 'european' 


\section{Fig. 10. Query for which the plan is to be constructed}

1. Step 1: The query involves two tables, CARS and BRANDS. The application of the operator CHECK_TABLES over the two relations results in the determination that the first is a hybrid one and the second a locally stored one.

2. Step 2: The operator CHECK_PEERS is applied to the catalog of peer $p_{1}$, in order to determine the peers of interest of the query. Taking into consideration the age of tuples found in relation CARS and the system catalog, the peer $p_{1}$ decides that the peers of interest are peers 2 and 8 .

3. Step 3: The operator CALL_WS is applied over each peer of interest.

4. Step 4: For each peer over which a CALL_WS is applied, we apply the operator WRAPPER_POP to coordinate the execution of its operations.

5. Step 5: The operator FILL is applied for the result of each WRAPPER_POP.

6. Step 6: The rest of the query plan is constructed as usual, with the only difference that the subtree of relation CARS is the one constructed in the previous steps.

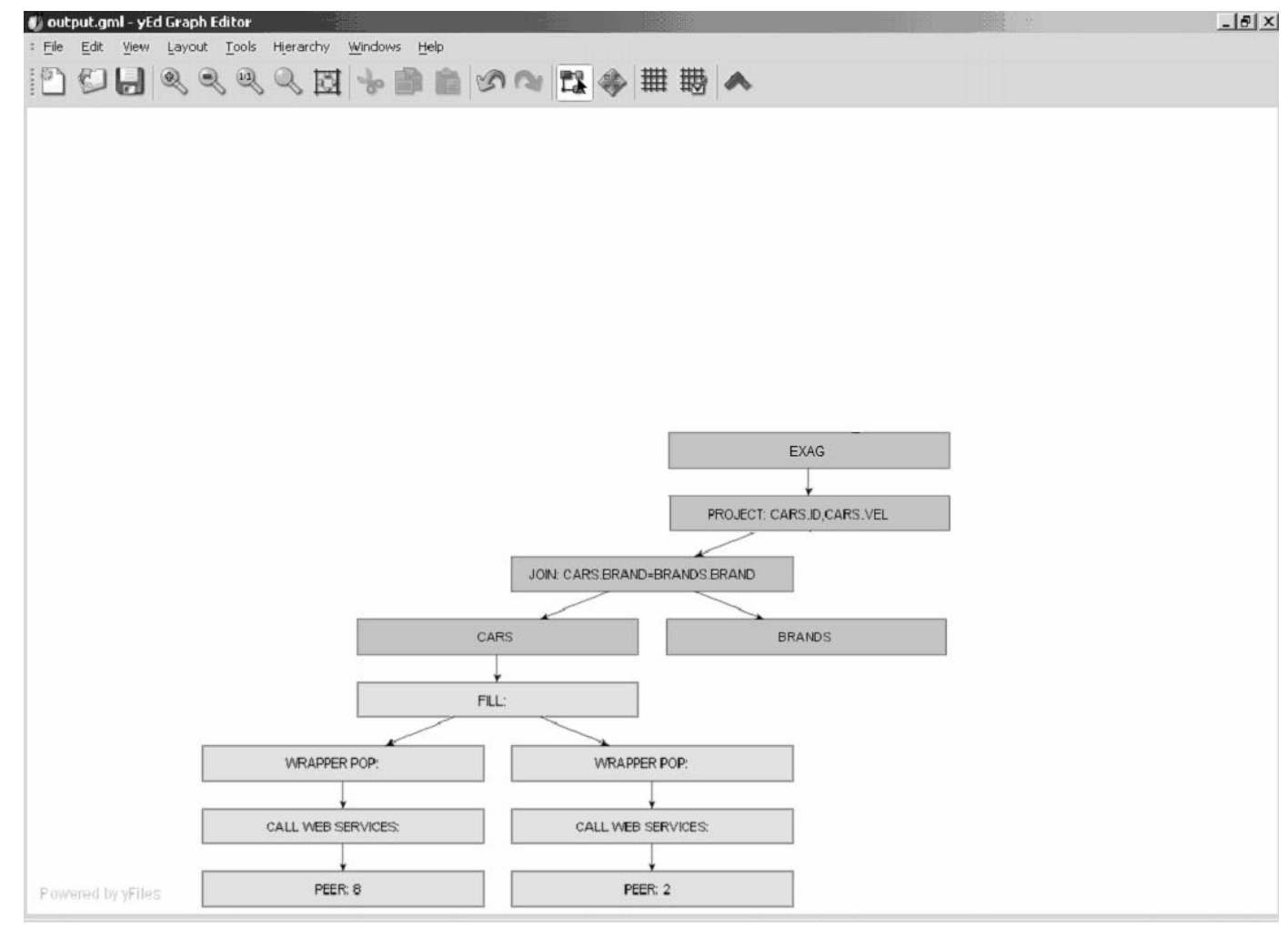

Fig. 11 Query plan for the aforementioned query of Fig. 10 


\section{IMPLEMENTATION}

Figure 12 shows the full-blown architecture required to support our approach for context-aware query processing in Ad-Hoc environments of peers. The elements shown in the figure are divided with respect to the client and the server roles played by peers. To play the client role, a peer comprises a traditional query processing architecture, involving a parser, an optimizer and a query processor. A local database and the system catalog complement the ingredients of the client part of a peer. Playing the server role amounts in publishing a set of web services, hosted by an application server which is responsible for their proper execution. As usually, whenever a query is posed, the parser is the first module that is fired. The optimizer produces alternative plans out of which the best, with respect to a given cost model, is chosen. The query execution engine executes the query over the local database and returns the results.

Our first prototype implementation does not currently support the query optimizer subsystem. Instead, standard query plans are produced after parsing the user queries. The query execution subsystem includes a mechanism that allows visualizing the aforementioned plans. Figure 11, gives a visualized execution plan through the Yed tool that graphically presents graphs.

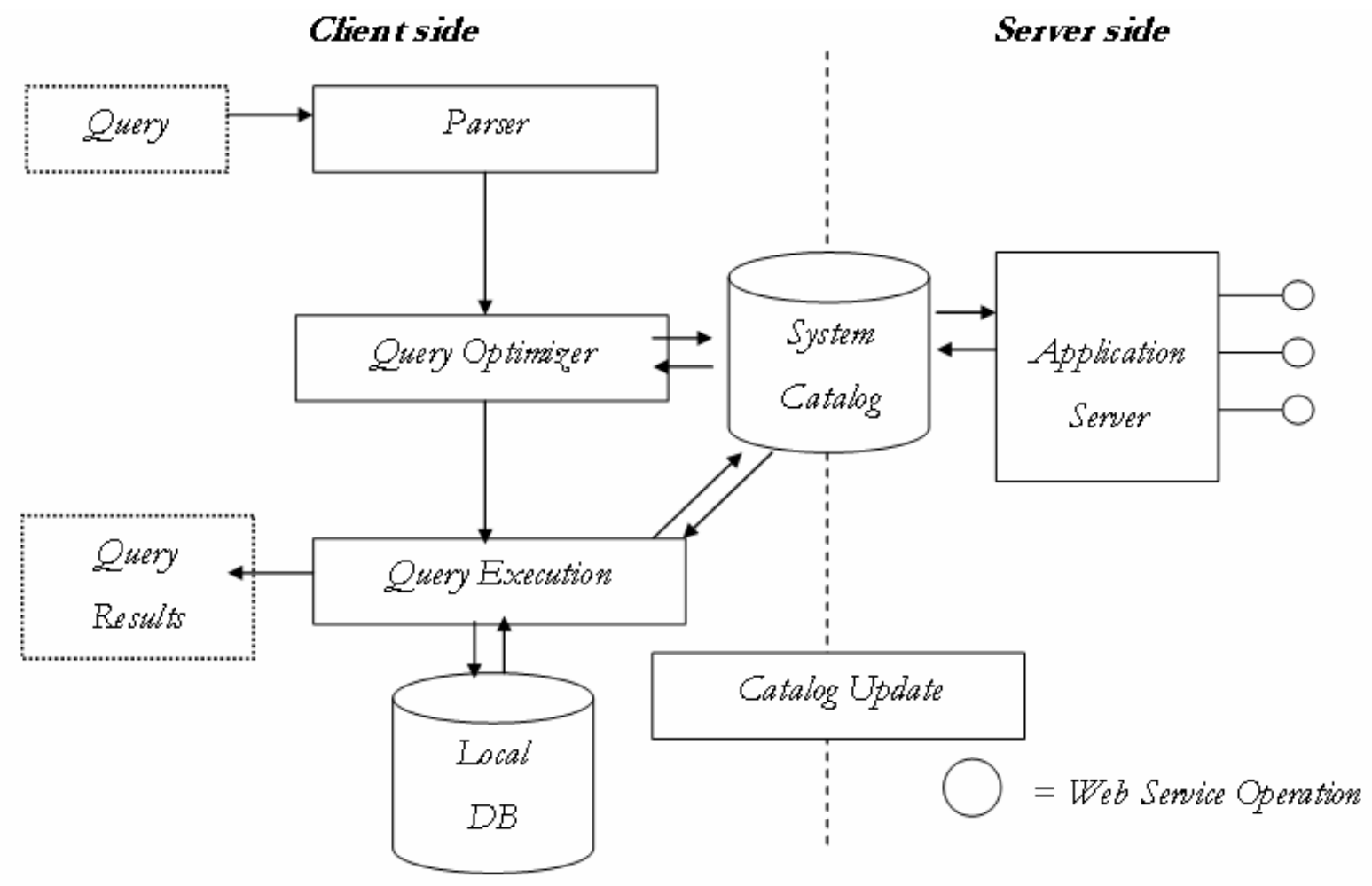

Fig. 12. System Architecture 
Populating and updating the contents of the system catalog is done either statically, or dynamically. In the former case, the peer is responsible for updating the catalog through a catalog-specific API. The static update of the catalog takes advantage of the possible availability of peer-specific dynamic service discovery mechanisms. Such mechanisms may be exploited by the peer itself which takes further charge of updating the catalog accordingly.

The dynamic catalog update is realized by the catalog update subsystem, which relies on WSAMI, a middleware platform for mobile web services (Issarny et al., 2005). WSAMI provides the Naming \& Directory service that allows the dynamic discovery of web services provided in mobile computing environments. Specifically, WSAMI is based on an SLP server -i.e., an implementation of the standard SLP (http://www.openslp.com) protocol-- for the discovery of networked entities in mobile computing environments.

\section{RELATED WORK}

The work that is closely related with the proposed approach for context-aware query processing over ad-hoc environments of peers can be categorized into work concerning the fundamentals of heterogeneous database systems, context-aware computing and approaches that specifically focus on context-aware service-oriented computing. The prominent approaches that fall in the aforementioned categories are briefly summarized in the remainder of this section.

\subsection{Heterogeneous Database Systems}

Our approach for querying of ad-hoc environments of peers bares some similarity with the traditional wrapper-mediator architectures used in heterogeneous database systems (Roth \& Schwarz, 1997), (Haas et al., 1997). Such systems consist of a number of heterogeneous data sources. The user of the system has the illusion of a homogeneous data schema which is actually realized by the wrapper-mediator architecture. In particular, each data source is associated with a wrapper. The wrapper encapsulates the data source under a well-defined interface that allows executing queries. Each user query is translated by the mediator into data source specific queries, executed by corresponding wrappers. As opposed to traditional heterogeneous database systems in the environments we examine the roles of users and data sources are not discrete. Each peer is a heterogeneous data source offering information to other peers that play the role of the user. Therefore, each peer may eventually serve as a data source and a user issuing queries. The analogous to the wrapper elements in our case is the web services that give access to peers playing the role of data sources. The analogous to the mediator element is the hybrid relation mapping procedure that executes workflows on web services. In simple words a traditional 
heterogeneous database system is a "1 mediator to $\mathrm{N}$ wrappers architecture". An ad-hoc environment of peers in our case is an " $\mathrm{N}$ mediator to $\mathrm{N}$ wrappers architecture".

Another fundamental difference between the environments we examine and traditional heterogeneous data base systems is that in our case the cardinality and the contents of the set of data sources may constantly change.

\subsection{Context-Aware Computing and Infrastructures}

In (Dey, 2001) context is defined as any information that can be used to characterize the interaction between a user and an application, including the user and the application. Several middleware infrastructures follow this definition toward enabling context-reasoning and management (Fahy \& Clarke, 2004), (Chen, Finin, \& Joshi, 2003), (Chan \& Chuang, 2003), (Capra, Emmerich, \& Mascolo, 2003), (Gu, Pung, \& Zhang, 2005), (Roman et al., 2002). Amongst these approaches CASS (Fahy \& Clarke, 2004) bares some similarity with our approach since context is modeled in terms of a relational data model. However, in our approach we do not assume centralized information management and virtual relations are dynamically compiled.

\subsection{Context-Aware Service-Oriented Computing}

In general, the integration of context-awareness and service-orientation just began to gain the attention of the corresponding research communities. In (Keidl \& Kemper, 2004), for instance, the authors introduce ways for associating context to web service invocations. In (Maamar, Mostefaoui, \& Mahmoud, 2005) the authors go one step further by examining the problem of customizing web service compositions with respect to contextual information. Web service execution is customized according to different types of context. Similarly, in (Zahreddine \& Mahmoud, 2005) the authors propose a framework for dynamic context-aware service discovery and composition. Specifically, contextual information regarding the technical characteristics of user devices is used towards discovering services that match these characteristics.

\section{CONCLUSIONS AND FUTURE WORK}

In this paper, we have dealt with context-aware query processing in ad-hoc peer-to-peer networks. Each peer in such an environment has a database over which users want to execute queries. This database involves (a) relations which are locally stored and (b) relations which are virtual or hybrid. In the case of virtual relations, all the tuples of the relation are collected from peers that are present in the network at the time when the query is posed. Hybrid relations involve both locally stored tuples and tuples collected from the network. The collaboration among peers is performed through web services. The integration of the external data, before they 
are locally collected to a peer's database is performed though a workflow of operations. To perform query processing in the traditional way, but rather, we involve context-aware query processing techniques that exploit the neighborhood of each peer and the web service infrastructure that deals with the heterogeneity of peers. In this setting, we have formally defined the system model for $S Q L^{P}$, an extension of traditional SQL on the basis of contextual environment requirements that concern the termination of queries, the failure of individual peers and the semantic characteristics of the peers of the network. We have precisely defined the semantics of the language $S Q L^{\mathrm{P}}$. We have also discussed issues of data integration, performed through workflows of web services. Moreover, we have presented an initial query execution algorithm as well as the typical definition of all the operators which can take place in a query execution plan. A prototype implementation that is implemented is also discussed.

\section{ACKNOWLEDGMENT}

This research is co-funded by the European Union - European Social Fund (ESF) \& National Sources, in the framework of the program "Pythagoras II" of the "Operational Program for Education and Initial Vocational Training" of the $3^{\text {rd }}$ Community Support Framework of the Hellenic Ministry of Education

\section{REFERENCES}

Abolhasan, M., Wysocki, T., \& Dutkiewicz, E. (2004). A review of routing protocols for mobile ad hoc networks. AdHoc Networks, 2, 1-22.

Androutsellis-Theotokis, S., \& Spinellis, D. (2004). A survey of peer-to-peer content distribution technologies. ACM Computing Sumeys, 36(4), 335-371.

Babcock, B., Babu, S., Datar, M., Motwani, R., \& Widom, J. (2002, June). Models and issues in data stream systems. In Proceedings of the $21^{\text {st }}$ ACM SIGACT-SIGMOD-SIGART Symposium on Principles of Database Systems (PODS'02), p. 1-16, Madison, Wisconsin, USA.

Capra, L., Emmerich, W., \& Mascolo, C. (2003). CARISMA: Context - Aware Reflective Middleware System for Mobile Applications. IEEE Transactions on Softwane Engineering, 29(10), p. 929-945.

Chan, A.T., \& Chuang, S.-N. (2003). MobiPADS: A Reflective Middleware for Context-Aware Mobile Computing. IEEE Transactions on Software Engineering, 29(10), p. 1072-1085.

Chen, H., Finin, T., \& Joshi, A. (2003). An Ontology for Context-Aware Pervasive Computing Systems. Knowledge Engineering Review, 18(3), 197-207.

Chlamtac, I., Conti, M., \& Liu, J. J.-N. (2003). Mobile ad hoc networking: imperatives and 
challenges. Ad Hoc Networks, 1(1), 13-64.

Dey, A. K. (2001). Understanding and Using Context. Personal and Ubiquitous Computing, 5(1), 4-7.

Fahy, P., \& Clarke, S. (2004, June). CASS - Middleware for Mobile Context-Aware Applications. In Proceedings of the 2nd ACM SIGMOBILE International Conference on Mobile Systems, Applications and Services (MobiSys'04), Boston, MA, USA.

Gu, T., Pung, H.-K., \& Zhang, D.-Q. (2005). A Service-Oriented Middleware for Building Context-Aware Services. Joumal of Network. and Computer Applications, 28, 1-18.

Haas, L.M., Kossmann, D., Wimmers, E. L., \& Yang, J. (1997, August). Optimiqing queries across diverse data sources. In Proceedings of 23rd International Conference on Very Large Data Bases (VLDB'97), p. 276--285, Athens, Greece.

Issarny, V., Sacchetti, D., Tartanoglou, F., Sailhan, F., Chibout, R., Levy, N., \& Talamona, A. (2005). Developing Ambient Intelligence Systems: A Solution Based on Web Services. Joumal of Automated Software Engineering, 12(1), p. 101-137.

Keidl, M., \& Kemper, A. (2004, March). A framework, for context-aware adaptable web services. In Proceedings of 9th International Conference on Extending Database Technology (EDBT '04), p. 826-829, Heraklion, Crete, Greece.

Maamar, Z., Mostefaoui, S., \& Mahmoud, Q. (2005, January). Context for Personalized Web Sernices. In Proceedings of 38th IEEE Hawaii International Conference on System Sciences (HICSS'05), p. 166.2, Big Island Hawaii, USA.

Madhavan, J., Bernstein, P. A., Doan, A., \& Halevy, A. Y. (2005, April). Corpus-based schema matching. In Proceedings of the 21st International Conference on Data Engineering (ICDE 2005), p. 57--68, Tokyo, Japan.

Ozsu, T., \& Valduriez, P. (1991). Principles of Distributed Database Systems. Prentice-Hall.

Roman, M., Hess, C. K., Cerqueira, R., Ranganathan, A., Campbell, R. H., \& Nahrstedt, K. (2002). Gaia: A Middleware Infrastructure to Enable Active Spaces. IEEE Pervasive Computing, 1(4), 74-83.

Roth, M. T., \& Schwarz, P. M. (1997, August). Don't scrap it, wrap it! A urapper architecture for legacy data sources. In Proceedings of 23rd International Conference on Very Large Data Bases (VLDB'97), p. 266-275, Athens, Greece.

Zahreddine, W., \& Mahmoud, Q. H. (2005, March). An agent-based approach to composite mobile web semices. In Proceedings of 19th International Conference on Advanced Information Networking and Applications (AINA 2005), p. 189-192, Taipei, Taiwan. 